\title{
$\mathrm{C}|\mathrm{E}| \mathrm{D}|\mathrm{L}| \mathrm{A} \mid \mathrm{S}$
}

Centro de Estudios

Distributivos, Laborales y Sociales

Maestría en Economía

Facultad de Ciencias Económicas

Universidad Nacional de la Plata

\section{Construcción de una Matriz de Contabilidad Social para Paraguay para el Año 2009}

Martín Cicowiez y Horacio Santander

Documento de Trabajo Nro. 164

Julio, 2014

ISSN 1853-0168 


\section{MINISTERIO DE HACIENDA}

\section{SUBSECRETARIA DE ESTADO DE ECONOMIA UNIDAD DEL SISTEMA DE INFORMACIÓN ECONÓMICA}

FINANCIERA

Documento de Trabajo

Construcción de una Matriz de Contabilidad Social para Paraguay para el Año $2009^{*}$

Martín Cicowiez +

Horacio Santander ${ }^{++}$

\footnotetext{
* El presente trabajo se realizó en el contexto del proyecto "Public Expenditure Review-Construction of a Dynamic Computable General Equilibrium (CGE) Model" para Paraguay del Banco Mundial. Los autores agradecen los comentarios de Carolina Díaz-Bonilla, Fritzi Koehler, Miguel Mora, Rodi Ozuna, César Rojas, Victor Ferreira y Julio Nuñez. Así mismo se agradece a todos los funcionarios del Ministerio de Hacienda y del Banco Central del Paraguay que colaboraron en la elaboración de los datos utilizados en la construcción de la MCS. La SAM resultante se encuentra disponible en <http://www.economia.gov.py/v2/>.

+ CEDLAS-UNLP; martin@depeco.econo.unlp.edu.ar

${ }^{++}$Ministerio de Hacienda; horacio_santander@hacienda.gov.py
} 


\section{Introducción}

En este documento se describe el procedimiento seguido para la construcción de una matriz de contabilidad social (SAM; del inglés Social Accounting Matrix) para Paraguay para el año 2009. El objetivo que se persigue es describir con detalle el trabajo realizado. Asimismo, la documentación de los pasos seguidos puede servir de base para futuras actualizaciones de la SAM elaborada.

La SAM que se describe en este trabajo será utilizada para calibrar MAMS (Maquette for MDG Simulations), un modelo de equilibrio general computable desarrollado en el Banco Mundial para evaluar distintas cuestiones relacionadas con el desarrollo económico. Sin embargo, la misma SAM puede utilizarse también para calibrar modelos de equilibrio general computable alternativos.

En el caso de Paraguay, no existen muchos antecedentes respecto de la construcción de matrices de contabilidad social. ${ }^{1}$ De hecho, sólo conocemos tres MCS elaboradas para Paraguay con anterioridad. En Molinas y Cabello (2004) se elaboró una MCS relativamente agregada (i.e., sólo identifica ocho sectores productivos clasificados por letra del CIIU) para el año 1998. Los autores utilizaron la MCS para calibrar el Modelo Estándar del IFPRI (Lofgren et al., 2002) a fin de evaluar diversos escenarios de liberalización comercial. Por otro lado, en Biedermann y Corvalán (2008) elaboraron una MCS para calibrar una versión anterior de MAMS; el modelo se utilizó para evaluar alternativas de financiamiento para el alcance de los Objetivos de Desarrollo del Milenio. Por último, en Ludena (2006) se describe el procedimiento utilizado para construir una MCS de Paraguay para el año 2004 para ser utilizada en la base de datos multi-país que elabora el Global Trade Analysis Project (GTAP). Por otro lado, existen algunas aplicaciones a Paraguay del análisis insumo-producto; ver Biedermann (2004) y Biedermann y Benítez (2009).

El resto del documento se organiza de la siguiente manera. La Sección 2 realiza una descripción resumida de las matrices de contabilidad social en general. La Sección 3 describe el procedimiento seguido para elaborar una SAM agregada para Paraguay para el año 2009. En la Sección 4 se muestra cómo se elaboró la SAM desagregada correspondiente. En particular, se desagregaron actividades y productos, y factores productivos. Asimismo, se describen todos los ajustes que fue necesario realizar. En la Sección 5 se expone el procedimiento de balanceo empleado para "ajustar" la matriz que se obtiene al combinar fuentes diversas de información y para distintos años. En la Sección 6 se describe la estructura económica de Paraguay a partir de la SAM elaborada. En la Sección 7 se describe la forma en

\footnotetext{
${ }^{1}$ La revisión de antecedentes está basada en el trabajo de Galeano Paredes (2010).
} 
que se realizaron las desagregaciones adicionales que requiere la calibración de la versión completa (i.e., versión ODM) de MAMS.

\section{La Matriz de Contabilidad Social: Conceptos Básicos}

La Tabla 2.1 resume las transacciones que captura una SAM. En pocas palabras, una SAM es una matriz cuadrada que registra todas las transacciones de una economía en un año determinado. Las filas y columnas de una SAM se denominan cuentas. Las cuentas que contiene una SAM pueden agruparse en actividades, productos (i.e., bienes y servicios), instituciones, y ahorro-inversión. Como puede observarse, la consistencia macro/microeconómica (i.e., oferta=demanda, ingresos=gastos, etc.) se asegura siempre que las sumas de filas y columnas correspondientes sean iguales.

Tabla 2.1: condiciones de equilibrio en la SAM

\begin{tabular}{|c|c|c|c|c|c|c|}
\hline & \multicolumn{6}{|c}{ gastos } \\
\cline { 2 - 7 } ingresos & firmas & hogares & gobierno & sav-inv & row & total \\
\hline firmas & & $\mathrm{C}$ & $\mathrm{G}$ & $\mathrm{I}$ & $\mathrm{E}$ & demanda \\
\hline hogares & $\mathrm{Y}$ & & & & $\mathrm{TR}$ & ingreso \\
\hline gobierno & & $\mathrm{T}$ & & & $\mathrm{TR}$ & ingreso gob \\
\hline sav-inv & & $\mathrm{SH}$ & $\mathrm{SG}$ & & $\mathrm{SF}$ & ahorro \\
\hline row & $\mathrm{M}$ & & & & & out forex \\
\hline total & oferta & gasto & gasto gob & inversión & in forex & \\
\hline
\end{tabular}

En lo que sigue se describe cada una de las condiciones de consistencia macro/microeconómica que deben cumplirse en una SAM; como se verá, es fácil verificar que las mismas se cumplen en el caso de la SAM elaborada para Paraguay. La oferta agregada (OA) se iguala con demanda agregada (DA). Analíticamente,

$$
\begin{aligned}
& O A=D A \\
& P I B P M+M=C+G+I+X
\end{aligned}
$$

donde PIB PM es el producto interno bruto a precios de mercado, $\mathrm{M}$ son las importaciones, $\mathrm{C}$ es el consumo privado, G es el consumo público, I es la inversión, y X son las exportaciones. A su vez, la 
inversión se descompone en formación bruta de capital fijo y variación de existencias. La Tabla 2.2muestra la composición del PIB de Paraguay para 2009, tal como se la registra en la SAM. ${ }^{2}$

Tabla 2.2: Composición del PIB - Paraguay 2009

\begin{tabular}{|l|r|r|}
\hline Agregado Macro & $\begin{array}{r}\text { Guaraníes } \\
\text { (miles mill.) }\end{array}$ & $\begin{array}{r}\text { Porcentaje } \\
\text { PIB }\end{array}$ \\
\hline Demanda Global & & \\
\hline Consumo Privado & 53,986 & 76.1 \\
\hline Inversión & 10,685 & 15.1 \\
\hline Variación Existencias & 227 & 0.3 \\
\hline Consumo Gobierno & 8,642 & 12.2 \\
\hline Exportaciones & 21,672 & 30.5 \\
\hline Total & 95,211 & \\
\hline Oferta Global & & \\
\hline PIB Precios Mercado & 70,980 & 100.0 \\
\hline Importaciones & 24,231 & 34.1 \\
\hline Total & 95,211 & \\
\hline \hline Fuente: SAM Paraguay 2009. & \\
\hline
\end{tabular}

Como se observa, el presupuesto público también aparece reflejado en la SAM. Así, el ahorro corriente del gobierno se define como

$$
S G=Y G-E G
$$

En el caso de Paraguay, las cuentas fiscales para 2009 reflejan un ahorro público corriente de 3.023 miles de millones de dólares, equivalentes a 4,3 puntos porcentuales del PIB.

La igualdad entre ahorro e inversión también aparece reflejada en la SAM. Es decir,

$$
\mathrm{I}=\mathrm{SH}+\mathrm{SG}+\mathrm{SF}
$$

donde SH es el ahorro privado, SF es el ahorro del resto del mundo, y SG es el ahorro del gobierno definido más arriba.

\footnotetext{
${ }^{2}$ Cabe hacer notar que los valores de exportaciones e importaciones de la Tabla 2.2 ya fueron ajustados para reflejar la presencia de re-exportaciones (ver más abajo). En cambio, los valores que se muestran más adelante en la Tabla 3.1 surgen directamente de las Cuentas Nacionales que elabora el BCP.
} 
La cuenta corriente de la balanza de pagos aparece reflejada en la SAM como

$$
E+T R+S F=M
$$

donde TR son las transferencias netas recibidas desde el resto del mundo.

Por último, el ingreso privado $(\mathrm{Y})$ se destina a consumir, ahorrar y pagar impuestos directos. Analíticamente,

$$
\mathrm{Y}=\mathrm{C}+\mathrm{T}+\mathrm{SH}
$$

donde T son los impuestos directos que enfrentan las familias.

La SAM de Paraguay para 2009 se elaboró en dos etapas, siguiendo el procedimiento que suele denominarse "de arriba hacia abajo" (ver Round (2003), Reinert y Roland-Holst (1997), entre otros). En primer lugar, se preparó una SAM agregada o MacroSAM que muestra, en forma de matriz, la información agregada que capturan las cuentas nacionales, la balanza de pagos, y la información fiscal. En segundo lugar, se utilizó información de distintas fuentes (ver más abajo) para desagregar los sectores productivos (i.e., actividades y productos), los factores primarios de producción, y los hogares.

\section{La MacroSAM}

Por construcción, la MacroSAM es consistente con los agregados macroeconómicos tal como aparecen en las cuentas nacionales. La MacroSAM así construida se muestra en la Tabla 3.1. Se eligió 2009 como año base por ser el último para el que se contaba con información al momento de iniciar el proceso de construcción de la SAM. Cabe aclarar, sin embargo, que resultaría relativamente fácil construir una matriz para un año más reciente como 2010. 
Tabla 3.1: MacroSAM Paraguay 2009

(miles de millones de guaraníes)

\begin{tabular}{|c|c|c|c|c|c|c|c|c|c|c|c|c|c|c|c|c|c|}
\hline & act & com & $f$-asal & f-noasal & f-cap & hhd & gov & row & tax-act & tax-com & tax-vat & tax-imp & tax-dir & tax-fac & $s-i$ & dstk & total \\
\hline act & & 133,039 & & & & & & & & & & & & & & & 133,039 \\
\hline $\mathrm{com}$ & 68,978 & & & & & 53,986 & 8,642 & 34,328 & & & & & & & 10,685 & 227 & 176,844 \\
\hline f-asal & 23,963 & & & & & & & 1,201 & & & & & & & & & 25,165 \\
\hline f-noasal & 13,213 & & & & & & & & & & & & & & & & 13,213 \\
\hline f-cap & 26,230 & & & & & & & 1,519 & & & & & & & & & 27,749 \\
\hline hhd & & & 24,145 & 13,213 & 24,944 & & 1,969 & 2,544 & & & & & & & & & 66,816 \\
\hline gov & & & & & 2,805 & 10 & & 33 & 655 & 1,492 & 4,450 & 977 & 2,193 & 1,019 & & & 13,634 \\
\hline row & & 36,886 & & & & & & & & & & & & & & & 36,886 \\
\hline tax-act & 655 & & & & & & & & & & & & & & & & 655 \\
\hline tax-com & & 1,492 & & & & & & & & & & & & & & & 1,492 \\
\hline tax-vat & & 4,450 & & & & & & & & & & & & & & & 4,450 \\
\hline tax-imp & & 977 & & & & & & & & & & & & & & & 977 \\
\hline tax-dir & & & & & & 2,193 & & & & & & & & & & & 2,193 \\
\hline tax-fac & & & 1,019 & & & & & & & & & & & & & & 1,019 \\
\hline$s-i$ & & & & & & 10,628 & 3,023 & $-2,739$ & & & & & & & & & 10,911 \\
\hline dstk & & & & & & & & & & & & & & & 227 & & 227 \\
\hline total & 133,039 & 176,844 & 25,165 & 13,213 & 27,749 & 66,816 & 13,634 & 36,886 & 655 & 1,492 & 4,450 & 977 & 2,193 & 1,019 & 10,911 & 227 & \\
\hline
\end{tabular}

REFERENCIAS: act $=$ actividades, com = productos, $f$-asal = trabajo asalariado, $f$-noasal = trabajo no asalariado, $f$-cap $=$ excedente de explotación bruto, hhd = hogares, gov = gobierno, row = resto del mundo, tax-act = impuestos sobre actividades, tax-com = impuestos sobre productos, tax-vat = impuesto al valor agregado, tax-imp = aranceles, tax-dir = impuestos directos, tax-fac = contribuciones a la seguridad social, s-i = ahorro-inversión, dstk = variación de existencias.

Como se verá, la mayor parte de la información que recoge la MacroSAM se obtuvo de las cuentas nacionales que elabora el Banco Central de Paraguay (BCP). En particular, se utilizaron las siguientes secciones $^{3}$, siempre para el total de la economía - la información sectorial se utilizó para construir la MicroSAM (ver más abajo):

- (3.2.1) valor bruto de la producción: consumo intermedio y producto interno bruto

- (3.4.3) producto interno bruto por tipo de gasto

- (3.5.12) generación del ingreso primario

- (3.5.49) impuestos a la producción

- (3.6.8)cuentas consolidadas de la nación

Adicionalmente, se utilizó (a) información fiscal elaborada por Dirección de Política Fiscal de la Subsecretaria de Estado de Economía del Ministerio de Hacienda para completar los ingresos y gastos

\footnotetext{
${ }^{3}$ La lista siguiente muestra, entre paréntesis, el número de tabla tal como aparece en la información que publica el BCP en su página web (ver <http://www.bcp.gov.py/>).
} 
corrientes del gobierno, y (b) información sobre transferencias corrientes obtenida de la cuenta corriente de la balanza de pagos.

En lo que sigue se describe la fuente de información utilizada para completar cada una de las celdas de la MacroSAM.

Celda [row,com], [com,hhd], [com,gov], [com,row], [com,s-i], [com,dstk]= las importaciones de bienes y servicios, el consumo total de los hogares (i.e., "consumo privado"), el consumo total del gobierno (i.e., "consumo público"), las exportaciones de bienes y servicios, la formación bruta de capital fijo, y la variación de existencias se obtuvieron directamente de la información sobre PIB por tipo de gasto de las cuentas nacionales.

Celda [com,act] = el consumo intermedio total se obtuvo directamente de las cuentas nacionales; en particular, del cuadro con información sobre valor bruto de la producción (VBP).

Celda [f-asal,act],[f-noasal,act], [f-cap,act] = los pagos a los factores productivos, trabajo asalariado (i.e., f-asal), trabajo no asalariado (i.e., f-noasal; ingreso mixto), y capital (i.e., f-cap) se obtuvieron directamente del cuadro de las cuentas nacionales que registra la generación del ingreso primario. Los pagos al factor capital se estimaron como la suma del "Excedente de explotación" y el "Consumo de capital fijo".

Celda [tax-act,act] = el cuadro mencionado en el párrafo anterior también brinda información sobre los "Otros impuestos a la producción". De hecho, las celdas de la MacroSAM mencionadas en el párrafo anterior sumadas a la celda aquí descripta conforman el valor agregado bruto.

Celda $[$ act,com] = la oferta total se obtuvo mediante la suma de las celdas que se explicaron en los párrafos anteriores.

Celda [tax-com,com], [tax-vat,com], [tax-imp]= la recaudación que generan estos tres impuestos (i.e., impuesto selectivo al consumo, impuesto al valor agregado, y los derechos de importación, en este orden) se obtuvo directamente de la información sobre impuestos a la producción de las cuentas nacionales. En el primer caso, se sumaron el "Impuesto selectivo al consumo" y los "Otros impuestos". ${ }^{4}$ En los demás casos, la información se tomó sin ninguna modificación.

\footnotetext{
${ }^{4}$ Cabe aclarar, sin embargo, que los "Otros impuestos" registrados en las Cuentas Nacionales son cero desde 2001.
} 
Celda [hhd,f-asal] $=$ el ingreso por trabajo asalariado de los hogares se estima a partir de dos componentes: (1) los salarios que pagan las actividades productivas, y (2) las "Remuneración de Asalariados procedentes del resto del mundo, netas" que se obtienen de las cuentas consolidadas de la nación que forman parte de las cuentas nacionales que elabora el BCP.

Celda [tax-fac,f-asal] = el contenido de esta celda se refiere a la contribución al fondo de jubilación contribuciones a la seguridad social. La información surge de los datos fiscales obtenidos de Dirección de Política Fiscal de la Subsecretaria de Estado de Economía del Ministerio de Hacienda.

Celda [hhd,f-cap] = el ingreso de capital de los hogares se estima como la diferencia entre el ingreso total del capital y el ingreso de capital que recibe el gobierno (ver más abajo). El ingreso total del capital incluye dos componentes: (1) los pagos al capital que realizan las actividades productivas, y (2) la "Renta de la propiedad y de la Empresa Procedente del resto del mundo, neta" que registran las cuentas consolidadas de la nación.

Celda [gov,f-cap] = el ingreso de capital que recibe el gobierno se calcula a partir de los siguientes tres componentes que registran las cuentas fiscales que elabora la Dirección de Política Fiscal de la Subsecretaria de Estado de Economía del Ministerio de Hacienda: Ingreso contractual p/ ItaipúYacyretá, Renta de Activos Financieros, y Otros Ingresos No Tributarios.

Celda [hhd,f-noasal] = el ingreso por trabajo no asalariado que reciben los hogares se calcula como el total de pagos al "ingreso mixto" que realizan las actividades productivas.

Celda [gov,hhd] = las transferencias desde los hogares hacia el gobierno se refieren a los "otros recursos corrientes" del gobierno que aparecen registrados en los datos fiscales obtenidos de Dirección de Política Fiscal de la Subsecretaria de Estado de Economía del Ministerio de Hacienda.

Celda [tax-dir,hhd] = el monto de impuestos directos que pagan los hogares corresponde a la suma de los impuestos "a la renta neta y utilidades" y "sobre el patrimonio" que aparecen registrados en los datos fiscales obtenidos de Dirección de Política Fiscal de la Subsecretaria de Estado de Economía del Ministerio de Hacienda.

Celda $[\mathbf{s - i , h h d ]}=$ el ahorro de los hogares se obtiene de manera residual como la diferencia entre sus ingresos y gastos. Lamentablemente, no contamos con una fuente de información alternativa para estimar el ahorro de los hogares. 
Celda [hhd,gov] = las transferencias desde el gobierno hacia los hogares se obtuvieron como residuo con el objetivo de mantener balanceada la cuenta del gobierno. Es decir, se supone que la información de los demás elementos que aparecen en la fila y columna del gobierno se conoce con más precisión. Cabe mencionar, sin embargo, que el monto de transferencias así estimado no varía de forma sustancial respecto del monto que reporta la información fiscal de la Dirección de Política Fiscal de la Subsecretaria de Estado de Economía del Ministerio de Hacienda.

Celda [s-i,gov] = el ahorro del gobierno se calculó como la diferencia entre los ingresos y gastos corrientes reportados en la información fiscal de la Dirección de Política Fiscal de la Subsecretaria de Estado de Economía del Ministerio de Hacienda.

Celda [f-asal,row] = el ingreso que el trabajo asalariado obtiene del resto del mundo corresponde a la "Remuneración de Asalariados procedentes del resto del mundo, netas" que aparece registrado en las cuentas consolidadas de la nación.

Celda [f-cap,row] = el ingreso que el capital obtiene del resto del mundo corresponde a la "Renta de la propiedad y de la Empresa Procedente del resto del mundo, neta" que registran las cuentas consolidadas de la nación.

Celdas[hhd,row] y [gov,row]= las transferencias desde el resto del mundo hacia los hogares y el gobierno se obtienen de combinar información de (1) las cuentas consolidadas de la nación que elabora el BCP, (2) la cuenta corriente de la balanza de pagos. Las primeras brindan el monto total de transferencias desde el resto del mundo a Paraguay. La segunda permite identificar la participación del sector privado y del gobierno en ese total.

Celda $[\mathbf{s - i , r o w ]}=$ el ahorro del resto del mundo (i.e., el negativo del saldo de la cuenta corriente de la balanza de pagos) se obtuvo por diferencia pero, por construcción, resulta igual al que registran las cuentas consolidadas de la nación. En este caso, cabe mencionar que existe una diferencia considerable entre el ahorro del resto del mundo calculado como se mencionó y el ahorro del resto del mundo que registra la cuenta corriente de la balanza de pagos (i.e., - $2,738,763$ millones de guaraníes en la MacroSAM versus $-330,920$ millones de guaraníes en la cuenta corriente de la balanza de pagos).En nuestro caso, a fin de lograr un mayor grado de consistencia con los agregados macroeconómicos, decidimos utilizar el valor de las cuentas nacionales como cierto. 


\section{La MicroSAM}

En términos generales, la MicroSAM o matriz de contabilidad social desagregada se elabora a partir de desagregar las celdas de la MacroSAM que contienen información referida a actividades y/o productos. Para ello, debido a que no se cuenta con tablas de insumo-producto actualizadas (i.e., para el año $2009)^{5}$, se utilizaron las siguientes fuentes de información complementaria:

- (3.5.10) PIB por tipo de ingreso desagregado por actividad CNAP

- (3.3.1) valor bruto de la producción por actividad CNAP

- (3.3.2) consumo intermedio por actividad CNAP

- impuestos a la producción (i.e., tax-com, tax-imp, tax-vat) por producto CNPP elaborada en la Unidad del Sistema de Información Económico Financiera de la Subsecretaría de Estado de Economía del Ministerio de Hacienda

- exportaciones e importaciones por producto CNPP elaborada en la Unidad del Sistema de Información Económico Financiera de la Subsecretaría de Estado de Economía del Ministerio de Hacienda

- tablas insumo-producto de 1997 (i.e., cuadros de oferta y utilización); de esta fuente de información se obtuvieron los coeficientes insumo-producto, la estructura por producto del consumo público y privado, la estructura por producto de las columnas de formación bruta de capital fijo y variación de existencias, la relación entre márgenes de distribución y oferta, y la estructura por producto del valor bruto de producción de las actividades

El primer paso para pasar de la MacroSAM a la MicroSAM consiste en elegir el nivel de desagregación que se desea utilizar. En particular, debe determinarse el número de actividades, productos, factores de producción (e.g., trabajo y capital), y sectores institucionales que aparecerán como cuentas individuales en la SAM desagregada. En nuestro caso, construimos una SAM con la mayor desagregación que permiten las cuentas nacionales. Así, se identifican 33 actividades y 46 productos, respectivamente (ver Tabla 4.1). ${ }^{6}$ Los factores primarios de producción se desagregan en tres: trabajo asalariado, trabajo no

\footnotetext{
${ }^{5}$ La disponibilidad de tablas insumo-producto actualizadas permite construir una MicroSAM con relativa facilidad.

${ }^{6}$ Sin embargo, la versión actual identifica 32 actividades y 45 productos, ya que, por inconsistencias entre los datos de comercio y los de producción, fue necesario agregar los productos químicos y farmacéuticos y de tocador.
} 
asalariado, y capital. ${ }^{7}$ Los impuestos son los mismos que los identificados en la MacroSAM. Por su parte, la inversión se desagrega en formación bruta de capital fijo y variación de existencias. Finalmente, se identifican tres instituciones: el gobierno, el resto del mundo y los hogares- que representan al sector privado completo.

Tabla 4.1: cuentas de la SAM Paraguay 2009

\begin{tabular}{|c|c|c|}
\hline Actividades (32) & Productos (45) & Productos (45) (cont.) \\
\hline Productos primarios (5) & Productos primarios (14) & Servicios (11) \\
\hline Agricultura & Algodón en rama & Electricidad y agua \\
\hline Ganadería & Caña de azúcar & Construcción \\
\hline Forestal & Otros cultivos industriales & Servicios de comercialización \\
\hline Pesca & Soja & Transportes \\
\hline \multirow[t]{2}{*}{ Minería } & Otros cereales & Correo y telecomunicaciones \\
\hline & Tubérculos & Servicios financieros y de seguros \\
\hline Manufacturas (16) & Frutas y otros productos agrícolas & Servicios de alquiler y de vivivenda \\
\hline Carnes & Ganado vacuno & Servicios a la empresa \\
\hline Aceites & Otros tipos de ganado & Servicios de restaurantes y hoteles \\
\hline Lácteos & Otros productos pecuarios & Servicios a los hogares \\
\hline Molin. Pan. & Productos forestales & Servicios gubernamentales \\
\hline Azúcar & Productos de la pesca & \\
\hline Otros alim. & Productos de la minería & Factores (3) \\
\hline Beb. Tab & & Trabajo asalariado \\
\hline Textiles & Manufacturas (21) & Trabajo no asalariado \\
\hline Cuero y calz. & Carne de vacuno & Capital \\
\hline Madera & Otras carnes & \\
\hline Papel & Aceites y grasas vegetales & Instituciones (3) \\
\hline Ref. petroleo y Químicos & Productos lácteos & Hogares \\
\hline Min. no metal. & Productos de molinería y panadería & Gobierno \\
\hline Metales & Azúcar & Resto del Mundo \\
\hline Maq. y equipo & Otros productos alimenticios & \\
\hline \multirow[t]{2}{*}{ Otros prod. } & Bebidas y tabaco & Impuestos (6) \\
\hline & Productos textiles y prendas de vestir & Actividades \\
\hline Servicios (11) & Cuero y productos del cuero & Valor agregado \\
\hline Elec. y agua & Madera y productos de la madera & Productos \\
\hline Const. & Productos del papel y cartón & Aranceles \\
\hline Comercio & Combustibles y lubricantes & Directos \\
\hline Transporte & Prod. químicos y Prod. farmaceuticos y de tocador & Contribuciones seguridad social \\
\hline Comunic. & Neúmaticos, cámaras y otros productos del caucho & \\
\hline Financieros & Productos minerales no metálicos & Ahorro-inversión (2) \\
\hline Alquilerviv. & Productos del hierro y no ferrosos & Ahorro-inversión \\
\hline Serv. Empresas & Productos metálicos estructurales & Variación de existencias \\
\hline Hoteles y restaur. & Maquinaria y equipo, aparatos domésticos y otros & \\
\hline Serv. Hogares & Vehículos automotores & \\
\hline Serv. gobierno & Otros productos manufacturados & \\
\hline
\end{tabular}

${ }^{7}$ En una etapa posterior, el capital se desagrega a fin de identificar al factor tierra. Por su parte, el trabajo se desagrega de acuerdo a su nivel de calificación. 
En lo que sigue se describe el procedimiento seguido para desagregar las celdas que contienen información referida a actividades y/o productos. Las demás celdas se toman directamente de la MacroSAM.

Celda [com,act] = el consumo intermedio se calculó a partir de combinar (a) el consumo intermedio total de cada actividad registrado en 2009, con (b) la composición por bien del consumo intermedio que surge de los coeficientes insumo-producto de la matriz de utilización del año 1997. Analíticamente,

$$
S A M_{c, a}=C I_{a}^{2009} \frac{\text { utilización } n_{c, a}^{1997}}{\sum_{c^{\prime}} \text { utilización } n_{c^{\prime}, a}^{1997}}
$$

donde c $\left(c^{\prime}\right)$ y a indexan productos y actividades, respectivamente, $S A M_{c, a}$ son las celdas de la MicroSAM para 2009 que registran el consumo intermedio, $C I_{a}^{2009}$ es el consumo intermedio total de la actividad a en 2009 , y utilizació $n_{c, a}^{1997}$ es el consumo intermedio que registra el cuadro de utilización de 1997. Como se mencionó, el valor de $C I_{a}^{2009}$ está disponible en las cuentas nacionales.

Celda [f-lab-asal,act], [f-lab-noasal,act], [f-cap,act] = la demanda factorial se desagregó entre actividades utilizando información disponible en las cuentas nacionales. En este caso, no fue necesaria la realización de ningún tipo de ajuste. Los factores productivos se desagregan en trabajo asalariado, trabajo no asalariado (i.e., ingreso mixto), y capital (i.e., la suma de excedente de explotación y consumo de capital fijo).

Celda [tax-act,act] $=$ los datos sobre producto interno bruto por tipo de ingreso de las cuentas nacionales reportan el monto de los impuestos a la producción que enfrenta cada una de las actividades productivas que identifican las cuentas nacionales.

Celda [act,com] = la matriz de oferta para 2009 se obtuvo a partir de combinar información de VBP (valor bruto de producción) por actividad (CNAP) para 2009 con la estructura que arroja la matriz de oferta de $1997 .^{8}$ Es decir, el VBP de cada actividad que reporta el BCP se desagregó utilizando la misma estructura por producto que la registrada en 1997. En general, existe una relación 1 a 1 entre actividades y productos de las cuentas nacionales; sólo en los casos de las actividades "agricultura" y "ganadería" existe un número de productos más grande que el número de actividades (i.e., 7/1 y 3/1 para agricultura y ganadería, respectivamente). Por otro lado, las proporciones registradas en 1997 no

\footnotetext{
${ }^{8}$ La matriz de oferta muestra la producción de cada producto proveniente de cada una de las actividades.
} 
varían de forma sustancial de las obtenidas a partir de información preliminar del BCP para 2009. Analíticamente,

$$
S A M_{a, c}=V B P_{a}^{2009} \frac{\text { of erta }_{a, c}^{1997}}{\sum_{c^{\prime}} \text { oferta } \operatorname{fer}_{a, c^{\prime}}^{1997}}
$$

dondeVBP $P_{a}^{2009}$ es el valor bruto de la producción de la actividad a en 2009, y of erta a,c $^{1997}$ es la matriz de oferta de las tablas insumo-producto de 1997.

Celda $[$ row,com] $=$ las importaciones por producto fueron estimadas a partir de establecer una concordancia entre productos registrados conforme al Clasificador Nacional de Productos del Paraguay (CNPP) en las tablas insumo-producto de 1997 y los datos de comercio exterior, obtenidos de la Dirección Nacional de Aduanas (DNA), clasificados de acuerdo a la Nomenclatura Común del Mercosur (NCM). Adicionalmente, se utilizó información de balanza de pagos para desagregar el comercio de servicios. Luego, la estructura de importaciones resultante se utilizó para desagregar el monto de importaciones registrado en la MacroSAM. Analíticamente,

$$
S A M_{\text {row }, c}=\operatorname{MacroSA} M_{\text {row }, \text { com }} \frac{\operatorname{impos}_{c}^{2009}}{\sum_{c^{\prime}} \operatorname{impos}_{c^{\prime}}^{2009}}
$$

dondeMacroSAM $M_{\text {row,com }}$ son las importaciones registradas en la MacroSAM, e $\operatorname{impos}_{c}^{2009}$ son las importaciones de producto c de 2009.

Celda [tax-com,com], [tax-vat,com], [tax-imp,com] = Los datos de los ingresos del Gobierno en concepto de impuestos se obtuvieron de la Subsecretaría de Estado de Tributación (SET) del Ministerio de Hacienda y la Dirección Nacional de Aduanas (DNA). La SET clasifica sus ingresos tributarios por actividad (CIIU 3), en tanto la DNA presenta los ingresos tributarios generados por el bien importado clasificados por NCM. ${ }^{9}$ A partir de esta información, se construyó una matriz de impuestos identificando sectorialmente los mismos de acuerdo al clasificador CNPP de las Cuentas Nacionales. Se identificaron los ingresos tributarios provenientes de los impuestos a los productos (impuesto al valor agregado, impuesto selectivo al consumo y los derechos de importación).

Celda [marg,com] = los márgenes de distribución correspondientes a cada producto se computaron a partir de las relaciones entre oferta doméstica e importada y margen de distribución registradas en

\footnotetext{
${ }^{9}$ Paraguay no aplica impuestos a las exportaciones.
} 
1997. En la MacroSAM los márgenes de distribución no aparecen registrados, al formar parte de la celda [act,com].

Celda [com,hhd] = el consumo por producto de los hogares se obtiene como el producto entre el consumo total registrado en la MacroSAM y la estructura por producto calculada a partir de la matriz de utilización de 1997. Analíticamente,

$$
S A M_{c, h h d}=\operatorname{MacroSA}_{c o m, h h d} \frac{\text { utilización } n_{c, h h d}^{1997}}{\sum_{c^{\prime}} \text { utilización } n_{c^{\prime}, h h d}^{1997}}
$$

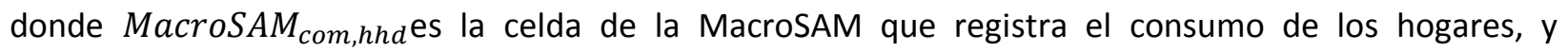
utilizació $n_{c, h h d}^{1997}$ es el consumo de los hogares por producto que aparece en la matriz de utilización de 1997.

Celda [com,gov] = el consumo por producto del gobierno también se obtiene como el producto entre el consumo total registrado en la MacroSAM y la estructura por producto calculada a partir de la matriz de utilización de 1997. Analíticamente,

$$
S A M_{c, g o v}=\operatorname{MacroSA}_{c o m, g o v} \frac{\text { utilización } n_{c, g o v}^{1997}}{\sum_{c^{\prime}} \text { utilización } n_{c^{\prime}, g o v}^{1997}}
$$

Celda $[$ com,row] = las exportaciones que registra la MacroSAM se desagregan por producto utilizando un procedimiento similar al descripto en el caso de las importaciones. Analíticamente,

$$
S A M_{c, \text { row }}=\operatorname{MacroSA}_{c o m, r o w} \frac{\operatorname{expos}_{c}^{2009}}{\sum_{c^{\prime}} \operatorname{expos}_{c^{\prime}}^{2009}}
$$

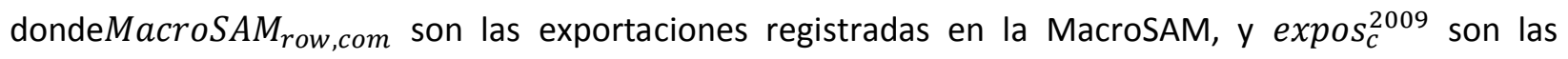
exportaciones de producto c de 2009.

Celda $[$ com,s-i] = la demanda de cada producto con destino inversión también se obtiene como el producto entre el consumo total registrado en la MacroSAM y la estructura por producto calculada a partir de la matriz de utilización de 1997. Analíticamente,

$$
S A M_{c, s-i}=\operatorname{MacroSA} M_{c o m, s-i} \frac{\text { utilizació } n_{c, s-i}^{1997}}{\sum_{c^{\prime}} \text { utilización } n_{c^{\prime}, s-i}^{1997}}
$$


Celda [com,dstk] = la variación de existencias por producto también se obtiene como el producto entre el consumo total registrado en la MacroSAM y la estructura por producto calculada a partir de la matriz de utilización de 1997. Analíticamente,

$$
S A M_{c, d s t k}=\operatorname{MacroSA} M_{c o m, d s t k} \frac{\text { utilización } n_{c, d s t k}^{1997}}{\sum_{c^{\prime}} \text { utilización } n_{c^{\prime}, d s t k}^{1997}}
$$

\section{Re-Exportaciones}

En este apartado se explica con algo de detalle la forma en que fueron computadas las reexportaciones.De acuerdo al Banco Central del Paraguay (BCP), las reexportaciones constituyen la reventa de productos importados a excursionistas receptivos en las zonas fronterizas entre Paraguay, Brasil (Ciudad del Este, Pedro Juan Caballero y Saltos del Guairá) y Argentina (Encarnación), principalmente. Son registradas como crédito en la cuenta bienes de la balanza de pagos.

Estas ventas fronterizas se ven favorecidas por dos factores. Por un lado, la existencia de una extensa frontera seca (incluye ciudades limítrofes separadas por avenidas divisorias) y fluvial de fácil traspaso en embarcaciones, entre Paraguay, Brasil y Argentina. Por otro lado, la política fiscal, cambiaria y comercial de Paraguay, que ha fomentado dichas transacciones buscando capturar recursos de la venta de productos con menores costos relativos e intensivos en tecnología provenientes de China (con intensidad en aumento en los dos últimos años), con capacidad de competir en el mercado interno de Brasil y Argentina.

El BCP estima el total de las reexportaciones considerando la siguiente metodología:

a) Desde cuarto trimestre del 2005, en coincidencia con la aplicación del régimen de turismo por Decreto Nro. 6.406/05 del 19 de setiembre del 2005, que establece la reducción del valor imponible de los bienes importados con finalidad de reexportación vía la venta a viajeros, se aumentó la registración de bienes importados en la Aduana hasta el 97\% por comparación con datos de las Aduanas del resto del mundo. Considerando esto, se ha estimado que el porcentaje de bienes susceptibles de reexportación es del $45 \%$ de las importaciones registradas, en tanto que la absorción domestica fue calculada en $30 \%$ de los bienes susceptibles de reexportación. Los datos en valores FOB son ajustados por Fletes y seguros (10\%), Aranceles (10\%), IVA (10\%) y Margen de comercialización (10\%), de manera acumulativa. 
b) Además, una parte correspondiente al comercio no registrado en la Aduana paraguaya, estimado por diferencia con las declaraciones de las Aduanas del resto del mundo, también se considera susceptible de ser reexportado. Desde cuarto trimestre del 2005 se considera una cobertura de la Aduana paraguaya con respecto a las Aduanas del resto del mundo del entre el $95 \%$ y $97 \%$, lo cual significa que se estima una importación no registrada del $5 \%$ al 3\% respectivamente, sustentado por el movimiento comercial entre las ciudades fronterizas del Paraguay conforme a las conclusiones de la divulgación titulada "Comparativo de registro con los socios del MERCOSUR y el resto del mundo", disponible en la web oficial del BCP.Las importaciones no registradas son ajustadas por fletes y seguros (10\%) y márgenes de comercialización (10\%), de manera acumulativa.

En base a la metodología descripta, el BCP estimó para el año 2009, unas reexportaciones por valor de 2.699 millones de dólares, representando el $46 \%$ del total de exportaciones del Paraguay. A partir de dicha cifra, se procedió a estimar las reexportaciones a nivel de productos del CNPP del SCN de Paraguay. Para ello, utilizando las distintas listas de excepción negociadas por Paraguay a nivel MERCOSUR, se identificaron a nivel de la NCM, aquellos productos importados en el 2009 y susceptibles de ser reexportados como ser: bebidas alcohólicas, perfumes, artículos deportivos, anteojos de sol, electrónicos, juguetes, entre otros.

Se identificaron los capítulos de la NCM a la cual pertenecen dichos ítems importados y su correspondencia con el CNPP, calculándose la suma total de dichas importaciones. Sobre esta estructura se estimaron las participaciones de dichos productos en el total importado susceptible de reexportación y se atribuyó dicho porcentaje a la reexportación a nivel de productos.

Al momento de utilizar la MCS elaborada para calibrar MAMS, las reexportaciones fueron sustraídas de las importaciones al mismo tiempo que se redujeron las exportaciones. Así, se mantuvo la igualdad entre suma de filas y columnas correspondientes. Alternativamente, podría modificarse el modelo para dar un tratamiento particular a las reexportaciones.

\section{El Balanceo de la SAM}

La matriz a la que se arriba siguiendo los pasos anteriores muestra desbalances entre sumas de filas y columnas correspondientes; algo esperable si tenemos en cuenta que se combinaron fuentes de información diferentes y para distintos años. En esta sección se describe el procedimiento seguido para balancear la MicroSAM obtenida. 
El problema de balanceo consiste en estimar la matriz $\mathrm{T}^{*}$ cercana a la matriz desbalanceada T pero que cumple con la restricción de igualdad entre sumas de filas y columnas correspondientes; es decir, debe cumplirse que

$$
\sum_{j} t_{i j}^{*}=\sum_{j} t_{j i}^{*}
$$

donde, en general, la transacción $t_{i j}^{*}$ será positiva siempre que la transacción correspondiente en la matriz original también sea positiva. El primer paso en el proceso de balanceo consiste en definir cómo se mide la distancia entre la matriz original T y la estimada $\mathrm{T}^{*}$. Luego, pueden agregarse distintos tipos de restricciones al problema de optimización que se resuelve (e.g., que cierto grupo de celdas sumen un determinado valor, que ciertas celdas tengan un límite inferior/superior dado, entre otras).

En nuestro caso, los desbalances se eliminaron utilizando el método de entropía cruzada (Robinson et al., 2001), que tiene la ventaja de emplear toda la información disponible en el proceso de estimación/balanceo de la SAM. Así, permite agregar restricciones sobre el contenido de ciertas celdas al proceso de minimización. Con este método, el problema de estimación se analiza en el contexto de la teoría de la información que Theil (1967) acercó a la teoría económica. En primer lugar, las celdas de la SAM se dividen por el total de transacciones a fin de que los elementos $t_{i j}$ puedan interpretarse como probabilidades, cuya suma es igual a la unidad. Analíticamente, se define

$$
a_{i j}=\frac{t_{i j}}{\sum_{i^{\prime}} \sum_{j^{\prime}} t_{i^{\prime} j^{\prime}}}
$$

En este caso, la noción de distancia entre $\mathrm{T}$ y $\mathrm{T}^{*}$ se escribe como la medida de entropía cruzada de Kullback-Leibler (1951) entre las nuevas $\left(a_{i j}^{*}\right)$ y viejas $\left(a_{i j}\right)$ probabilidades. Matemáticamente, el problema de optimización puede escribirse como

$$
\min =I\left(A^{*}: A\right) \sum_{i} \sum_{j} a_{i j}^{*} \ln \left(\frac{a_{i j}^{*}}{a_{i j}}\right)=\sum_{i} \sum_{j} a_{i j}^{*} \ln a_{i j}^{*}-\sum_{i} \sum_{j} a_{i j}^{*} \ln a_{i j}
$$

\section{s. a.}

$$
\sum_{j} a_{i j}^{*}=\sum_{j} a_{j i}^{*} \quad \forall i
$$




$$
\begin{aligned}
& \sum_{i} \sum_{j} a_{i j}^{*}=1 \\
& 0 \leq a_{i j}^{*} \leq 1
\end{aligned}
$$

La ecuación (5.2) impone como restricción que la suma de columna y filas correspondientes sea igual. Naturalmente, si todas menos una de las restricciones que impone (5.2) se están cumpliendo, la restante también se estará cumpliendo, de forma análoga a la Ley de Walras.

Adicionalmente, el procedimiento descripto permite agregar restricciones sobre conjuntos de celdas de la SAM; por ejemplo, puede imponerse que la suma de las celdas que representan el consumo de las familias replique el agregado macro de consumo privado. Analíticamente, se definen una matriz $\mathrm{G}$ de dimensión $\mathrm{n}$ x $\mathrm{n}$ que contiene unos para las celdas cuya suma desea imponerse como restricción, y ceros en caso contrario. Asumiendo que se imponen $k$ restricciones de este tipo, al conjunto de restricciones (5.2)-(5.4) se agrega (5.5),

$$
\sum_{i} \sum_{j} g_{i j}^{(k)} t_{i j}=\gamma^{(k)}
$$

donde $g_{i j}$ es un elemento de la matriz $\mathrm{G} \mathrm{y} \gamma^{(k)}$ es el valor que desea imponerse como restricción de agregación. En consecuencia, esta forma de actualizar la SAM permite incorporar toda la información que se posee para el año que se considera como base, no sólo las sumas de filas y columnas. ${ }^{10}$

En nuestro caso, se impusieron las siguientes restricciones, algunas obtenidas de la macro SAM y otras de los datos desagregados que se conocen con certeza: (1) los agregados macroeconómicos deben mantenerse inalterados respecto de los valores en la MacroSAM, (2) las exportaciones e importaciones de cada producto se mantienen en los valores computados a partir de información elaborada para 2009(es decir, neteadas de las reexportaciones y exportaciones no registradas), (3) los niveles de producción para algunos agregados sectoriales se mantienen en los valores conocidos en base datos de cuentas nacionales, y (4) la recaudación tributaria por impuestos indirectos también se mantiene en los valores computados a partir de información obtenida para 2009.

\footnotetext{
${ }^{10}$ Además, a la ecuación (5.1) pueden agregarse errores de estimación que reflejan la incertidumbre del analista con respecto a las distintas fuentes de información empleadas para construir la SAM original T (ver Robinson et al., 2001).
} 


\section{Descripción de la SAM}

En esta sección se realiza una descripción de la información registrada en la SAM. Con ella, se facilitará la interpretación de los resultados de las simulaciones de CGE que se planea realizar. En términos generales, se presentan las características de la estructura económica que son importantes para determinar los resultados de un modelo de CGE.

La intensidad de uso factorial en cada actividad productiva (e.g., relaciones sectoriales capital/trabajo) se muestra en la Tabla 6.1. La primera columna muestra la participación de cada sector en el valor agregado total expresado a precios corrientes. Por su parte, las demás columnas muestran cómo se distribuye el valor agregado sectorial entre los tres factores de producción identificados. En términos de la estructura económica, el 25,3\% del valor agregado total lo genera la actividad agroalimenticia. La intensidad factorial de cada actividad permite anticipar factores ganadores y perdedores de un shock que beneficie a unos sectores por sobre otros. En términos de valor, los sectores agrícola y minero son relativamente intensivos en tierra y activos del subsuelo, respectivamente. Por su parte, las actividades Administración pública, Salud y Educación son servicios relativamente intensivos en el empleo calificado. $^{11}$

\footnotetext{
${ }^{11}$ Sin embargo, cabe aclarar que el SCN 93 indica que las actividades públicas deben mostrar un excedente de explotación nulo, por lo que no se registran pagos a los stocks de capital público cuando las actividades producen bienes que se proveen de manera gratuita.
} 
Tabla 6.1: composición del valor agregado

(en porcentaje)

\begin{tabular}{|c|c|c|c|c|c|}
\hline Actividad & VAshr & $\begin{array}{r}\text { Labor, } \\
\text { Salaried }\end{array}$ & $\begin{array}{r}\text { abor, Non. } \\
\text { Salaried }\end{array}$ & Capital & Total \\
\hline Agricultura & 13.1 & 20.9 & 54.2 & 24.9 & 100.0 \\
\hline Ganadería & 6.4 & 22.7 & 6.5 & 70.7 & 100.0 \\
\hline Forestal & 1.8 & 3.2 & 1.6 & 95.1 & 100.0 \\
\hline Pesca & 0.1 & 0.0 & 100.0 & 0.0 & 100.0 \\
\hline Minería & 0.2 & 41.9 & 4.8 & 53.2 & 100.0 \\
\hline Carnes & 4.0 & 5.2 & 0.3 & 94.6 & 100.0 \\
\hline Aceites & 0.5 & 18.9 & 0.1 & 81.0 & 100.0 \\
\hline Lácteos & 0.2 & 44.2 & 1.2 & 54.6 & 100.0 \\
\hline Molin. Pan. & 0.5 & 54.8 & 7.0 & 38.2 & 100.0 \\
\hline Azúcar & 0.2 & 61.1 & 0.0 & 38.9 & 100.0 \\
\hline Otros alim. & 0.3 & 33.6 & 4.2 & 62.2 & 100.0 \\
\hline Beb. Tab & 2.3 & 28.6 & 0.1 & 71.3 & 100.0 \\
\hline Textiles & 1.5 & 40.8 & 45.9 & 13.3 & 100.0 \\
\hline Cuero y calz. & 0.3 & 71.9 & 12.1 & 16.0 & 100.0 \\
\hline Madera & 0.7 & 54.4 & 12.4 & 33.2 & 100.0 \\
\hline Papel & 0.6 & 73.4 & 3.8 & 22.9 & 100.0 \\
\hline Químicos & 0.6 & 66.0 & 1.0 & 33.0 & 100.0 \\
\hline Min. no metal. & 1.0 & 39.4 & 6.8 & 53.8 & 100.0 \\
\hline Metales & 0.4 & 59.7 & 5.3 & 35.0 & 100.0 \\
\hline Maq. y equipo & 0.7 & 60.1 & 16.1 & 23.8 & 100.0 \\
\hline Otros prod. & 0.7 & 45.1 & 24.2 & 30.7 & 100.0 \\
\hline Elec. y agua & 1.7 & 27.3 & 0.0 & 72.7 & 100.0 \\
\hline Const. & 7.3 & 49.4 & 17.0 & 33.6 & 100.0 \\
\hline Comercio & 20.0 & 23.9 & 31.6 & 44.5 & 100.0 \\
\hline Transporte & 4.4 & 57.5 & 15.6 & 26.9 & 100.0 \\
\hline Comunic. & 3.5 & 33.4 & 0.0 & 66.6 & 100.0 \\
\hline Financieros & 3.5 & 24.5 & 0.0 & 75.5 & 100.0 \\
\hline Alquiler viv. & 1.2 & 0.0 & 0.0 & 100.0 & 100.0 \\
\hline Serv. Empresas & 2.9 & 17.9 & 16.3 & 65.8 & 100.0 \\
\hline Hoteles y restaur. & 1.2 & 47.9 & 35.5 & 16.6 & 100.0 \\
\hline Serv. Hogares & 6.4 & 34.3 & 43.7 & 22.0 & 100.0 \\
\hline Serv. gobierno & 11.8 & 100.0 & 0.0 & 0.0 & 100.0 \\
\hline Total & 100.0 & & & & \\
\hline \multicolumn{2}{|c|}{ Fuente: SAM Paraguay 2009.} & & & & \\
\hline
\end{tabular}

Como veremos, los shocks que se originan en el resto del mundo se transmiten a la economía doméstica a través de la cuenta corriente de la balanza de pagos. Por lo tanto, es importante conocer de antemano la estructura del comercio internacional de Paraguay tal como se la captura en el modelo de CGE (ver 
Tabla 6.2). El sector agroindustrial en su conjunto representó en 2009 el 58,5\% del total exportado (ver columna EXPshr), con exportaciones que rondan el 26,2de su producción (ver columna EXP-OUTshr). Los sectores más orientados a la importación son Vehículos, Combustibles y lubricantes y Maquinaria y equipocon76,9, 70,8 y 61,6por ciento de su consumo cubierto con importaciones, respectivamente (ver columna IMP-DEMshr). 
Tabla 6.2: estructura del comercio internacional (en porcentaje)

\begin{tabular}{|c|c|c|c|c|}
\hline Producto & EXPshr & EXP-OUTshr & IMPshr & IMP-DEMshr \\
\hline Algodón en rama & 0.4 & 21.7 & 0.0 & 4.5 \\
\hline Caña de azúcar & 0.0 & 0.0 & 0.0 & 0.0 \\
\hline Otros cultivos industriales & 0.9 & 41.8 & 1.7 & 69.0 \\
\hline Soja & 15.8 & 64.5 & 0.3 & 4.4 \\
\hline Otros cereales & 10.0 & 73.3 & 0.4 & 15.3 \\
\hline Tubérculos & 0.0 & 0.0 & 0.1 & 0.6 \\
\hline Frutas y otros productos agrícolas & 1.5 & 21.5 & 0.6 & 11.2 \\
\hline Ganado vacuno & 0.0 & 0.0 & 0.1 & 0.4 \\
\hline Otros tipos de ganado & 0.0 & 0.0 & 0.1 & 2.0 \\
\hline Otros productos pecuarios & 0.1 & 1.1 & 0.1 & 2.2 \\
\hline Productos forestales & 0.7 & 10.2 & 0.0 & 0.0 \\
\hline Productos de la pesca & 0.0 & 0.2 & 0.1 & 23.1 \\
\hline Productos de la minería & 0.1 & 6.2 & 0.3 & 21.7 \\
\hline Carne de vacuno & 11.5 & 27.4 & 0.0 & 0.0 \\
\hline Otras carnes & 0.8 & 6.4 & 0.1 & 1.1 \\
\hline Aceites y grasas vegetales & 13.2 & 67.0 & 0.3 & 5.4 \\
\hline Productos lácteos & 0.1 & 2.2 & 0.2 & 7.3 \\
\hline Productos de molinería y panadería & 1.3 & 16.7 & 1.2 & 18.7 \\
\hline Azúcar & 0.5 & 11.4 & 0.0 & 0.5 \\
\hline Otros productos alimenticios & 0.1 & 2.1 & 2.9 & 39.4 \\
\hline Bebidas y tabaco & 0.6 & 2.2 & 0.6 & 3.4 \\
\hline Productos textiles y prendas de vestir & 1.1 & 8.0 & 4.3 & 29.0 \\
\hline Cuero y productos del cuero & 0.8 & 26.8 & 0.5 & 17.9 \\
\hline Madera y productos de la madera & 1.2 & 13.3 & 0.1 & 2.0 \\
\hline Productos del papel y cartón & 0.1 & 0.9 & 3.2 & 34.7 \\
\hline Combustibles y lubricantes & 0.0 & 0.0 & 20.3 & 98.6 \\
\hline Productos químicos & 1.3 & 17.1 & 18.9 & 73.0 \\
\hline Neúmaticos, cámaras y otros productos del caucho & 1.1 & 34.7 & 4.4 & 69.9 \\
\hline Productos minerales no metálicos & 0.1 & 1.3 & 2.2 & 22.3 \\
\hline Productos del hierro y no ferrosos & 0.4 & 11.5 & 2.8 & 51.3 \\
\hline Productos metálicos estructurales & 0.0 & 0.5 & 0.6 & 8.5 \\
\hline Maquinaria y equipo, aparatos domésticos y otros & 0.4 & 20.9 & 10.9 & 80.6 \\
\hline Vehículos automotores & 0.2 & 16.5 & 10.7 & 88.3 \\
\hline Otros productos manufacturados & 0.1 & 2.3 & 3.2 & 32.5 \\
\hline
\end{tabular}


Tabla 6.2: estructura del comercio internacional - cont. (en porcentaje)

\begin{tabular}{|c|c|c|c|c|}
\hline Electricidad y agua & 0.6 & 5.2 & 0.0 & 0.0 \\
\hline Construcción & 0.0 & 0.0 & 0.0 & 0.0 \\
\hline Servicios de comercialización & 0.0 & 0.0 & 0.0 & 0.0 \\
\hline Transportes & 4.0 & 10.1 & 5.0 & 19.4 \\
\hline Correo y telecomunicaciones & 0.4 & 2.2 & 0.1 & 0.6 \\
\hline Servicios financieros y de seguros & 0.7 & 3.8 & 0.9 & 7.7 \\
\hline Servicios de alquiler y de vivivenda & 0.0 & 0.0 & 0.0 & 0.0 \\
\hline Servicios a la empresa & 21.0 & 74.3 & 0.2 & 3.3 \\
\hline Servicios de restaurantes y hoteles & 5.0 & 31.1 & 2.0 & 23.9 \\
\hline Servicios a los hogares & 0.0 & 0.0 & 0.0 & 0.0 \\
\hline Servicios gubernamentales & 4.0 & 8.3 & 0.4 & 1.6 \\
\hline Total & 100.0 & 14.4 & 100.0 & 20.0 \\
\hline
\end{tabular}

Referencias: EXPshr = participación sector en total expos; EXP-OUTshr = participación expos en producción; IMPshr\% = participación sector en total impos; IMP-DEPshr = participación impos en consumo.

Según información de la SAM, en 2009 el gobierno de Paraguay recaudó impuestos y contribuciones a la seguridad social por un valor equivalente a 15,2\% del PIB (Tabla 6.3). De ese total, 20,3\% corresponde a impuestos directos, 9,5\% a contribuciones a la seguridad social, y el resto corresponde a impuestos indirectos.

Tabla 6.3: recaudación tributaria

\begin{tabular}{|l|r|r|r|}
\hline & $\begin{array}{r}\text { miles mill. } \\
\text { guaraníes }\end{array}$ & $\begin{array}{r}\text { porcentaje } \\
\text { recaudación }\end{array}$ & $\begin{array}{r}\text { porcentaje } \\
\text { Impuesto }\end{array}$ PIB \\
\hline Actividades & 655 & 6.1 & 0.9 \\
\hline Valor agregado & 4,450 & 41.3 & 6.3 \\
\hline Productos & 1,492 & 13.8 & 2.1 \\
\hline Aranceles & 977 & 9.1 & 1.4 \\
\hline Directos & 2,193 & 20.3 & 3.1 \\
\hline Factores $\left(^{*}\right)$ & 1,019 & 9.5 & 1.4 \\
\hline Total & 10,786 & 100.0 & 15.2 \\
\hline \hline
\end{tabular}

$(*)$ son contribuciones seguridad social

Fuente: SAM Paraguay 2009.

\section{Desagregaciones Adicionales para MAMS}

En primer lugar, la formación bruta de capital fijo se desagregó entre pública y privada; para ello, se utilizó información de Cuentas Nacionales suministrada especialmente por el BCP. Luego, tanto el 
consumo corriente como de capital del gobierno se desagregó en los siguientes sectores: educación, salud, agua y saneamiento, otra infraestructura, administración pública. Los datos necesarios se obtuvieron a partir de información elaborada del Sistema de Información Contable (SICO) del Ministerio de Hacienda para el año 2009. Esta información se muestra en la Tabla 7.1.

Tabla 7.1: estructura gasto del gobierno, corriente y de capital; en miles de millones de guaraníes; 2009

\begin{tabular}{l|r|r|r|r|}
\hline & \multicolumn{2}{|c|}{ Corriente } & \multicolumn{2}{c|}{ Capital } \\
\hline Sector & G\$ & porc & G\$ & porc \\
\hline c-edupgov & 1,647 & 17 & 47 & 3 \\
\hline c-edusgov & 757 & 8 & 42 & 2 \\
\hline c-edutgov & 464 & 5 & 52 & 3 \\
\hline c-saludgov & 2,207 & 22 & 197 & 11 \\
\hline c-wtsn & 36 & 0 & 49 & 3 \\
\hline c-otrinfra & 398 & 4 & 1,069 & 58 \\
\hline c-admpub & 4,349 & 44 & 376 & 21 \\
\hline total & 9,857 & 100 & 1,833 & 100 \\
\hline Fuente: elaboración propia en base al Sistema de \\
Información Contable (SICO) del Ministerio de \\
Hacienda; Año 2009.
\end{tabular}

Cabe hacer notar que, según información sobre comercio internacional de servicios, aproximadamente 9\% de la producción de servicios gubernamentales se exporta. En la desagregación por productos del consumo público total se supuso que $9 \%$ de la producción de cada servicio público se exporta. Ciertamente, este supuesto podría modificarse por otro más plausible, siempre que la información necesaria se encuentre disponible. Además, por falta de información, se supone que la estructura de costos de todas las actividades públicas es idéntica.

En esta etapa se supone que la composición por producto de la demanda para inversión es idéntica para todos los sectores públicos.

La desagregación de los pagos al factor trabajo por nivel de calificación se realiza empleando información de la EPH para el período 2004-2010. En particular, se utiliza la información de la Tabla 7.2 estableciendo una concordancia entre las actividades productivas que allí aparecen y las consideradas en la matriz de contabilidad social que se construyó. 
Tabla 7.2: participación categorías de trabajo en total masa salarial; por actividad; en porcentaje

\begin{tabular}{|l|r|r|r|r|}
\hline Sector & f-labn & f-labs & f-labt & total \\
\hline agric-otrprim & 89.9 & 7.7 & 2.4 & 100.0 \\
\hline ind-lowtec & 64.3 & 29.1 & 6.6 & 100.0 \\
\hline otrind & 51.9 & 34.9 & 13.1 & 100.0 \\
\hline const & 73.6 & 17.0 & 9.4 & 100.0 \\
\hline comercio-hotel-rest & 51.0 & 37.8 & 11.1 & 100.0 \\
\hline elect-gas-agua-transp-comunic & 41.7 & 37.3 & 21.0 & 100.0 \\
\hline finanzas-svcprofesionales & 14.3 & 27.8 & 57.9 & 100.0 \\
\hline admpub & 15.8 & 41.6 & 42.6 & 100.0 \\
\hline educ-salud & 7.8 & 45.6 & 46.7 & 100.0 \\
\hline svcpersonales & 49.5 & 39.7 & 10.8 & 100.0 \\
\hline svcdomestico & 83.6 & 16.1 & 0.3 & 100.0 \\
\hline total & 53.0 & 29.1 & 17.9 & 100.0 \\
\hline f-labn = menos que secundaria completa & & & \\
\hline f-labs = menos que terciaria completa & & & \\
\hline f-labt = terciaria completa & & & & \\
\hline Fuente: elaboración propia en base a EPH $2004-2010$. & & \\
\hline
\end{tabular}

\section{Comentarios finales}

Este documento detalla los pasos seguidos para elaborar una matriz de contabilidad social para Paraguay para el año 2009. Como se mencionó, la SAM construida identifica 33 actividades y 46 productos del Sistema de Cuentas Nacionales de Paraguay.

Para adecuar la SAM al modelo MAMS, la formación bruta de capital fijo se desagregó entre pública y privada; para ello, se utilizó información de Cuentas Nacionales suministrada especialmente por el BCP. Luego, tanto el consumo corriente como de capital del gobierno se desagregó en los siguientes sectores: educación, salud, agua y saneamiento, otra infraestructura, administración pública. Los datos necesarios se obtuvieron a partir de información elaborada del Sistema de Información Contable (SICO) del Ministerio de Hacienda para el año 2009. De esta forma, para fines del modelo MAMS la SAM 2009 de Paraguay identifica 37 actividades y 51 productos. La SAM resultante se encuentra disponible en http://www.economia.gov.py/v2/. 


\section{Referencias}

Biedermann, Gustavo (2004). Análisis Sectorial de la Economía Paraguaya: Métodos y Aplicaciones Insumo-Producto. Banco Central del Paraguay.

Biedermann, Gustavo y Jorge Corvalán (2008). Paraguay. En R. Vos, E. Ganuza, H. Lofgren, M. V. Sanchez y C. Díaz-Bonilla. Políticas Públicas para el Desarrollo Humano: ¿Cómo Lograr los Objetivos de Desarrollo del Milenio en América Latina y el Caribe. Uqbar Editores.

Biedermann, Gustavo y Walter Benítez Melgarejo y Gustavo Biedermann (2009) Impacto de una Caída en la Demanda Externa sobre el PIB: Una Aplicación Insumo-Producto. Banco Central del Paraguay.

Galeano Paredes, Juan José (2010). Construcción de una Matriz De Contabilidad Social Agregada para Paraguay. Mimeo.

Kullback, Solomon y Richard A. Leibler (1951).On Information and Sufficiency. Annals of Mathematical Statistics 22 (1): 79-86.

Lofgren, Hans, Rebecca Lee Harris y Sherman Robinson (2002).A Standard Computable General Equilibrium (CGE) Model in GAMS. International Food Policy Research Institute (IFPRI) Microcomputers in Policy Research 5.

Ludena, Carlos (2006). GTAP 7 Data Base Documentation - I-O Tables: Paraguay. GTAP 7 Data Base Documentation.Center for Global TradeAnalysis.

Reinert, Kenneth A. y David W. Roland-Holst (1997). Social Accounting Matrices. En J. F. Francois y K. A. Reinert (eds.). Applied Methods for Trade Policy Analysis: A Handbook. Cambridge University Press.

Robinson, Sherman, AndreaCattaneo y Moataz El-Said (2001). Updating and Estimating a Social Accounting Matrix Using Cross Entropy Methods. Economic System Research 13 (1): 47-64.

Round, Jeffrey (2003).Constructing SAMs for Development Policy Analysis: Lessons Learned and Challenges Ahead.Economic Systems Research 15 (2): 161-183.

Theil, Henri (1967). Economics and Information Theory. Amsterdam: North-Holland. 